\title{
Effect of Coronavirus Disease 2019 on Patients with Bladder Cancer in China
}

\author{
Xiao Yang ${ }^{a}$ Juntao Zhuang ${ }^{a}$ Hao Yu $^{a}$ Qiang Cao ${ }^{a}$ Kai Li $^{a}$ Zijian Zhou ${ }^{a}$ \\ Jie Han ${ }^{a}$ Jiancheng $\mathrm{Lu}^{\mathrm{a}}$ Baorui Yuan ${ }^{\mathrm{a}}$ Qikai Wu ${ }^{\mathrm{a}}$ Dexiang Feng ${ }^{\mathrm{a}}$ \\ Lingkai Cai $^{a}$ Haiwei Yang ${ }^{a}$ Min Gu ${ }^{b}$ Pengchao Lia Qiang Lu ${ }^{a}$ \\ aDepartment of Urology, The First Affiliated Hospital of Nanjing Medical University, Nanjing, PR China; \\ ${ }^{b}$ Department of Urology, The Second Affiliated Hospital of Nanjing Medical University, Nanjing, PR China
}

\section{Keywords}

Bladder cancer · Progression · Hematuria · Telehealth ·

Coronavirus disease 2019

Dear Editor,

The coronavirus disease-2019 (COVID-19) pandemic in early 2020 has caused great global damage to healthcare systems. The Chinese government announced a stop to travel in and out of Wuhan, Hubei Province, on January 23,2020 , to control the spread of the virus. As of July 14,2020 , the number of confirmed cases globally has increased to 12,964,809 [1]. Defeating COVID-19 requires joint efforts worldwide.

Hematuria often occurs as an early symptom of bladder cancer. However, most people do not pay attention to this early warning sign, especially during a pandemic. Here, we demonstrate the effect of COVID-19 on patients with bladder cancer in China.

We collected information on patients with hematuria or suspected bladder tumors in the Urology Department of our hospital from January to May in 2019 and 2020. Data of patients hospitalized are summarized in Table 1, and patients with cystoscope are summarized in Table 2.

A total of 180 patients in 2019 and 155 patients in 2020 were included in this analysis. In 2020, the number of pa- tients with recurrence increased $(p=0.027)$ and highgrade urothelial carcinoma accounted for more cases $(p=0.044)$. The number of patients who underwent radical cystectomy was significantly higher than that in 2019 $(p=0.039)$. The average weekly number of cystoscopies in 2020 decreased significantly $(p=0.001)$.

Cystoscopy visits decreased because of the strict management of hospital visits and patients refusing invasive tests. However, cystoscopy is essential for bladder disease, especially early time. Deferred diagnosis and treatment can risk to tumor progression and recurrence [2]. We propose that patients seek help from doctors in time if symptoms occur, such as hematuria and urinary frequency. However, patients should not forget to practice selfprotection during the pandemic period.

The degree of tumor invasion affects whether conservative methods are adopted. In our hospital, more patients chose radical cystectomy in 2020. This may result from cancer progression. However, the final method depends on the patient's own decision. Considering the demand for regular intravesical instillations of chemother-

Xiao Yang, Juntao Zhuang, Hao Yu, Qiang Cao contributed equally to this work.
Qiang Lu or Pengchao Li 
Table 1. Data of patients hospitalized

$\begin{array}{lll}2019, & 2020, & p \text { value } \\ n=180 & n=155\end{array}$

\section{Gender, $n(\%)$ \\ Male \\ Female}

Age, years

Home address, $n$ (\%)

Nanjing

Outside-Nanjing

New/recurrence, $n$ (\%)

New

Recurrence

Treatment, ${ }^{\mathrm{a}} n(\%)$

TURBT

RC

Patients with operation

Time from admission to first operation, days

Days in hospital, days

Pathological grade, $n(\%)$

UMLMP

Low-grade urothelial carcinoma

High-grade urothelial carcinoma

Pathological staging in RC patients, $n$ (\%)

Muscle invasive

Nonmuscle invasive

Pathological staging in RC patients without NAC, $n$ (\%)

Muscle invasive

Nonmuscle invasive

Operations average week, $n$

TURBT average week, $n$

RC average week, $n$

$$
\begin{array}{r}
146(81.1) \\
34(18.9) \\
64.49 \pm 12.49 \\
70(38.9) \\
110(61.1) \\
121(67.2) \\
59(32.8) \\
121(82.3) \\
26(17.7)
\end{array}
$$

\section{$4.54 \pm 2.85$ \\ $9.19 \pm 6.78$}

$10(8.7)$

$43(37.4)$

$62(53.9)$

$19(65.5)$

$10(34.5)$

$$
15(60)
$$

$10(40)$

$7.85 \pm 3.86$

$6.20 \pm 2.93$

$1.45 \pm 1.32$

$\begin{array}{rr}119(76.8) & 0.330 \\ 36(23.2) & 0.547 \\ 63.67 \pm 12.46 & 0.255\end{array}$

$104(67.1)$

$86(55.5)$

$69(44.5)$

$92(71.9)$

$36(28.1)$

$4.50 \pm 2.80 \quad 0.890$
$8.78 \pm 5.34$

$8.78 \pm 5.34 \quad 0.571$

$2(1.9)$

0.044

$36(33.3)$

$70(64.8)$

$22(59.5)$

$15(40.5)$

$22(61.1)$

$14(38.9)$

$7.44 \pm 3.11$

$5.17 \pm 1.79$

$2.06 \pm 1.73$

0.615

0.930

0.725

0.204

0.230

TURBT, transurethral resection of bladder tumor; RC, radical cystectomy; UMLMP, urothelial neoplasm of malignant potential; NAC, neoadjuvant chemotherapy. ${ }^{\text {a }}$ Treatment: this data was only compared between TURBT and RC.

Table 2. Data of patients with cystoscope

\begin{tabular}{lccr}
\hline & $\begin{array}{l}2019, \\
n=568\end{array}$ & $\begin{array}{l}2020, \\
n=338\end{array}$ & $p$ value \\
\hline $\begin{array}{l}\text { Gender, } n(\%) \\
\quad \text { Male }\end{array}$ & $409(72.0)$ & $250(74.0)$ & 0.522 \\
$\quad$ Female & $159(28.0)$ & $88(26.0)$ & \\
Age, years & $61.82 \pm 13.87$ & $62.30 \pm 13.31$ & 0.610 \\
Biopsy rate, \% & $25.7(146 / 568)$ & $22.5(76 / 338)$ & 0.276 \\
Positive rate of biopsy, \% & $45.9(67 / 146)$ & $55.3(42 / 76)$ & 0.185 \\
Hospitalization rate, \% & $8.3(47 / 568)$ & $10.9(37 / 338)$ & 0.180 \\
Cystoscope average week, $n$ & $31.56 \pm 8.71$ & $17.79 \pm 13.09$ & 0.001 \\
$\quad$ From January to May & $30.33 \pm 8.22$ & $9.80 \pm 8.46$ & $<0.001$ \\
$\quad$ From January to March & $33.23 \pm 8.25$ & $24.00 \pm 11.08$ & 0.024 \\
$\quad$ From March to May & & & \\
\hline
\end{tabular}


apy drugs after transurethral resection of bladder tumors, patients are reluctant to visit hospitals repeatedly during this stressful period.

Bladder cancer requires long-term monitoring. Our department began online consultations since early March. A study shows $84.7 \%$ of patients prefer online consultations because this virus may do damage to human urogenital system [3]. Up to now, COVID-19 pandemic is still urgent abroad, and it may even last until 2025 [4]. The AGILE group in Italy demonstrates that it is even a decrease in patients with urgent urological treatment [5]. Thus, it is important to conduct telehealth and integrate it into traditional medical treatment, which is conducive to establishing a hierarchical medical system. However, the process of telehealth is too complicated for old patients to adopt. Simplifying the process and increasing promotion of it may be more useful for the elderly. For urologists, EAU20 has gone virtual this summer, which encourages urologists to adapt to online learning, as well as to telehealth.

Finally, we hope doctors pay attention to patients visiting with visible hematuria after the pandemic. It is possible that bladder cancer was not detected and that early symptoms were ignored during the pandemic.

\section{Conflict of Interest Statement}

The authors have no conflicts of interest to declare.

\section{Funding Sources}

This work was supported by the National Natural Science Foundation of China (Grant Nos. 81772711, 82073306, and 82072832), Jiangsu Province's Key Provincial Talents Program (ZDRCA2016006).

\section{Author Contributions}

Q. Lu and P.C. Li have given substantial contributions to the conception or the design of the manuscript, all authors to acquisition, analysis, and interpretation of the data, and X. Yang, J.T. Zhuang, and H. Yu to statistical analysis. X. Yang, J.T. Zhuang, H. $\mathrm{Yu}$, and $\mathrm{Q}$. Cao have participated to drafting the manuscript. Q. $\mathrm{Lu}$ and P.C. Li revised it critically. All authors read and approved the final version of the manuscript.

\section{References}

1 WHO. Coronavirus disease (COVID-19) Situation Report - 176.

2 Wallis CJD, Novara G, Marandino L, Bex A, Kamat AM, Karnes RJ, et al. Risks from deferring treatment for genitourinary cancers: a collaborative review to aid triage and management during the COVID-19 pandemic. Eur Urol. 2020 Jul;78(1):29-42.
3 Boehm K, Ziewers S, Brandt MP, Sparwasser $\mathrm{P}$, Haack M, Willems F, et al. Telemedicine online visits in urology during the COVID-19 pandemic-potential, risk factors, and patients' perspective. Eur Urol. 2020 Jul;78(1): $16-20$.

4 Kissler SM, Tedijanto C, Goldstein E, Grad $\mathrm{YH}$, Lipsitch M. Projecting the transmission dynamics of SARS-CoV-2 through the postpandemic period. Science. 2020;368(6493): $860-8$.
5 Porreca A, Colicchia M, D'Agostino D, Amenta M, Corsaro A, Zaramella S, et al. Urology in the time of coronavirus: reduced access to urgent and emergent urological care during the coronavirus disease 2019 outbreak in Italy. Urol Int. 2020;104(7-8):631-6. 